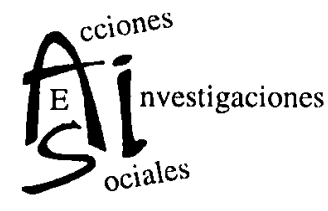

\title{
Génesis y esencia de las redes
}

\author{
MARIAN LEO IRANZO
}

ABOGADO

DIPLOMA DE ESPECIALIZACIÓN EN DIRECCIÓN DE ORGANIZACIONES DE ECONOMÍA SOCIAL

UNIVERSIDAD DE ZARAGOZA

Existen multitud de organismos con la palabra "red" en su denominación, no todos ellos tienen han nacido ni tienen la esencia de las redes de que aquí hablamos. Es necesario retomar esta esencia como iniciativa de la sociedad civil de progresar y resolver los conflictos, o afrontar las carencias que encuentra en su seno cuando los poderes "ad hoc" no lo hacen, lo intentan sin conseguirlo o crean y perpetúan todo ello. Una red es la conexión de "conciencias"dejando fluir a través suyo información y conocimientos en un movimiento de auto-apoyo y redistribución del poder; es la manera más activa de la sociedad viva de reivindicar el derecho y el deber de participar activamente en la consecución de su destino.

Palabras clave: Información, contactos y recursos, intercambio de conocimientos, auto-ayuda, enriquecimiento mutuo, sociedad civil, movimientos sociales, "tridimensionalidad", redistribución de poder. 


\section{Génesis y esencia de las redes}

Marian Leo Iranzo

\section{Introducción}

Durante siglos, nos hemos organizado y dirigido con estructuras semejantes a la pirámide. Desde el ejército romano a la iglesia católica, a los organigramas de General Mottors y la IBM, el poder y la comunicación han fluido de modo ordenado de la cima de la pirámide hasta su base.

La estructura piramidal o jerárquica ha sido defendida y criticada aunque sus detractores tardaron en encontrar un mejor modo de organización, los intentos fueron muchos. En el mundo de la dirección de organizaciones es obligado hablar de Douglas McGregor que introdujo una perspectiva humanista de la motivación en el trabajo a la que se denominó Teoría Y.

Las últimas décadas del pasado siglo vivimos en un mundo bullente de cambios, estos nos llevaron a la demolición de la pirámide. Resulta simple decir que existieron determinados factores en el nacimiento de las redes y más intentar aislarlos ya que la interrelación que se da entre ellos y la cadena causa-consecuencia es un todo en el que es difícil aislar elementos, aún así es necesario para conocer el espíritu de la red. Podemos analizar cada una de la causas a su vez interrelacionadas con las demás así como su repercusión en el mundo de redes.

- En primer lugar asistimos al paso de una sociedad industrial a una sociedad de la información donde las rígidas estructuras jerárquicas apenas encajan. Sorprende que tantas personas y organizaciones se resistan a la idea de una economía basada en la información y que, a pesar de 
una gran cantidad de evidencias, nieguen que la era industrial haya acabado. En palabras de Alvin Toffler "No se trata de una idea... es una realidad'.Nuestra vida está basada hoy en la generación y distribución de información, incluso podemos ir más allá y decir que nos encontramos en la sociedad del conocimiento

Una de grandes atracciones de la formación de redes es que es un modo fácil de obtener información. La red tiene como una de sus finalidades compartir información y contactos, pero las redes pueden ir mas lejos, a la creación e intercambio de conocimientos. Para ello es necesario que alguien en la red recoja nueva información y la sintetice llegando así a nuevas ideas. La red difunde estas ideas y pensamientos recién forjados. William de Bogart escribe al respecto. "Cada nuevo pensamiento se integra en el si guiente, produciendo una comprensión acumulativa de la naturaleza humana y del mundo en que vivimos. Estos nuevos modelos mentales son compartidos dentro de las recién formadas redes en todo el mundo".

Vivimos enterrados en una sobrecarga informativa que se genera y transmite a nuestro alrededor continuamente. Con ayuda de las redes, podemos seleccionar y adquirir únicamente aquella información que precisemos con la mayor celeridad posible. Las redes cortan diagonalmente las instituciones que albergan información y ponen a los individuos en contacto directo con la persona o recurso que buscan.

- Es segundo lugar asistimos a la destrucción de las jerarquías. Las instituciones centralizadas de la sociedad cuya existencia descansaba sobre jerarquías comenzaron a derrumbarse (al menos en lo que conocemos como mundo occidental). En su lugar surgen unidades descentralizadas, más pequeñas, uniéndose de modo informal unas con otras, y por tanto descansando mucho menos sobre estructuras formales. Las consideradas redes tradicionales tales como la familia, la iglesia... con un componente jerárquico, están perdiendo fuerza, lo cual crea un vacío en el que aparecen las nuevas redes como el equivalente espontáneo del momento actual de la antigua tribu, siendo esta de carácter más horizontal. Las jerar- 
quías han perpetuado incluso creado los problemas de los seres humanos, lejos de resolverlos lo cual tiene amplia relación con las dos causas siguientes.

- La necesidad de contacto. Los estudios acerca de la reacción de las personas a la tecnología, demuestran que lo que ocurre cuando una nueva tecnología es introducida en la sociedad, debe producirse una respuesta humana para "contrapesarla", o la tecnología será rechazada. En las últimas décadas se ha introducido mucha tecnología en nuestra sociedad.La naturaleza fría e impersonal de la jerarquía burocrática molesta cada vez mas a los individuos. En la red se busca mayor comunicación interpersonal, más contacto en respuesta a la frialdad de las instituciones existentes, el individuo reivindica su lado humano y la red satisface su necesidad de pertenencia. El fracaso de las jerarquías del que hablábamos en el punto anterior fuerza al individuo a buscar a otros fuera de sus organizaciones y de las instituciones y este es el primer paso para la creación de una red; personas o grupos de estas que se comunican y se enfrentan a sus problemas y preocupaciones. Cuanta más tecnología introducimos en nuestra sociedad, más individuos se congregarán, querrán estar con otros individuos.

- Existe una relación intima entre las dos causas expuestas anteriormente y esta: la autoayuda. Poco a poco empezamos a liberarnos e nuestra dependencia institucional colectiva, aprendiendo a confiar y depender de nosotros mismos. Nos apartamos de las instituciones que nos defraudan y comenzamos a aprender que tenemos la capacidad de actuar por nuestra cuenta. El fracaso del "estado del bienestar" -sin haber legado a su apogeo-, extendida la convicción entre los individuos de una sociedad de que ni este ni todas las instituciones existentes solucionarán sus problemas, hace que el individuo busque fuera de estos apoyo para resolverlos, lo primero es detectar intereses coincidentes, objetivos similares. Las redes aparecen cuando las personas tratan de cambiar la sociedad sin importar cual sea la causa, los objetivos o creencias, no importa dentro de que tipo de movimiento se inscriba ( social, político, religioso...) Los individuos se organizan y 
cambian aspectos de la sociedad, surgiendo una forma no burocrática pero muy efectiva de estructura organizativa. Las redes de autoayuda se sostienen por si mismas mas que por aportaciones externas, no tienen líderes profesionales, son abiertas (es decir, no hay unos requisitos estrictos para entrar en ellas), locales, innovadoras, desprovistas de ideología y persiguen un mayor grado de auto conciencia y una vida más plena y más libre. Este tipo de organizaciones demuestran este potencial oculto incluso en los miembros más vulnerables de la sociedad.

Las redes son en definitiva entre otras cosas un elemento de transformación social, cada revolución significa el cambio de manos del poder. Con las redes el poder puede quedar distribuido por todo el tejido social. A pesar de todas las proclamaciones de apoyarse en iniciativas de acción popular, la política tradicional se ha aplicado siempre desde arriba; son políticos economistas, estadistas quienes deciden las diferentes cuestiones, y pasan luego las consignas a todo el cuerpo de votantes A medida que van resultando evidentes los beneficios inherentes a la conexión y cooperación, comenzaron a aparecer redes para tratar de conseguir todo tipo de objetivos imaginables. Unas se centran en el desarrollo personal, en la búsqueda espiritual, en la reinserción de sus miembros; otras se ocupan e temas económicos y sociales. Algunas persiguen con fuerza intereses específicos de determinados grupos, y ejercen presión política por medios bastante convencionales, son las más vulnerables a la tentación de convertirse en organizaciones jerárquicas convencionales.

Sea cual sea su objetivo manifiesto, la función de la mayoría de estas redes es ofrecer apoyo y enriquecimiento mutuo, robustecer al individuo y cooperar en la transformación. La mayoría aspiran a un mundo más hospitalario. Por la riqueza de oportunidades de mutua ayuda y apoyo, la red presenta reminiscencias de su antecesor el sistema parental. No obstante "la familia" en este caso se basa en valores y convicciones profundamente compartidos, que resultan ser lazos más fuertes que la sangre.

Las redes son la estrategia de grupos pequeños para transformar la sociedad. Gandhi usó este tipo de coaliciones para conducir a la India a su independencia. El las llamaba "unidades 
en grupo", y las consideraba esenciales para el éxito. "El circulo de unidades así agrupadas de forma conveniente va a ir cre ciendo en circunferencias hasta que al final abarque al mundo entero". A comienzos del siglo pasado Edward Carpenter profetizó ese entrelazamiento y solapamiento de redes llamadas a crear "la sociedad acabada y libre".

Podriamos llegar a una conclusión de todo lo expuesto hasta ahora. Las jerarquías permanecen; nuestra creencia en su eficacia no.

El principio de las redes llega cuando como individuos asumimos nuestros problemas y dificultades y nos unimos a otros con la intención de así resolverlos.

«Las redes son un instrumento poderoso para la actuación social. Aquellos que cambiarían el mundo empezaron a hacerlo localmente, en grupos de personas de mentalidad similar con un propósito ideológico sencillo»

John Naisbitt

El movimiento feminista, por ejemplo, apareció siguiendo el modelo de un red clásica. En todas las partes de Inglaterra las mujeres se unieron en pequeños grupos para robustecer sus conceptos sobre si mismas y en consecuencia cambiar la tradicional posición de las mujeres en la sociedad. Los movimientos ecologistas y pacifistas de las últimas décadas del siglo XX comenzaron también en forma de redes.

Las redes son por lo general el primer momento de un grupo comunitario autónomo. Actualmente el modelo de redes tiende a sustituir a al forma jerárquica que queda asociada a frustraciones, despersonalización, inercia y fracaso.

\section{2. ¿Qué es una red?}

Sencillamente expuesto, las redes son individuos hablando unos con otros, compartiendo ideas, información y recursos. Lo importante no es la propia red, el producto acabado, sino el proceso para llegar a él: Las "conferencias, Ilamadas telefónicas, viajes, libros, periódicos, folletos, amigos mutuos, boletines..." Marilyn Ferguson.

Las redes sirven para fomentar la auto-ayuda, para intercambiar información, para hacer evolucionar a la sociedad, para 
mejorar la productividad y la vida del trabajo y para compartir los recursos. Están estructuradas para transmitir información de un modo más rápido, con más alto contacto y mayor eficiencia energética que cualquier otro proceso que conocemos.

"Las redes son la sociología apropiada -el equivalente a humano a la tecnología apropiada- que proporciona una forma de comunicación e interacción conveniente para el futuro del comienzo de esa década y más adelante, con escasez de energía y riqueza de información"

J. Lipnack y J. Stamps

Existen miles de redes similares, así como cientos de miles, a las que todos pertenecemos -las redes informales de amigos, grupos de discusión, organizaciones comunitarias- que nunca alcanzan la etapa organizativa.

Una indicación de la popularidad de la idea del establecimiento de redes son los muchos y variados grupos que incluyen la palabra red en sus nombres sin que por ello podamos identificar a todos ellos como redes en el sentido del que de ellas estamos hablando aquí.

Algunas redes se han convertido en organizaciones estables, activas. Otras siguen siendo fluidas y abiertas; otras desaparecen mientras sus miembros reaparecen en otras partes para crear otras redes. Existe incluso una red en la formación de redes: "La Consciousness Syntehsis Clearing House" de Redondo Beach, California, que ha elaborado una nueva teoría del proceso de formación de redes y desarrollando las perspectivas desde las que contemplar este fenómeno.

\section{Su estructura}

Virgina Hine las llamó Spin "redes polifacéticas segmenta das" "una red de pescar mal tejida con una multitud de nódulos o células de tamaños variados, cada un de ellas unida a las demás directa o indirectamente". Mientras que un esquema organizativo convencional muestra los diversos recuadros nítidamente enlazados entre sí.

En los movimientos sociales, esas células o nudos son grupos locales formados por un puñado de miembros o hasta por cientos de personas, Muchos se forman para cumplir una única tarea específica, y hoy están y mañana desaparecen. 
Cada segmento de un SPIN es autosuficiente, no se puede destruir un red a base de destruir a uno solo de sus líderes u órganos vitales. El centro, el corazón de la red está en todas partes. La debilidad de un burocracia se mide por su punto más débil. En una red hay muchas personas que pueden asumir las funciones de los demás. Esta característica recuerda la de la plasticidad del cerebro, que permite un solapamiento en sus funciones, de manera que las células dañadas puedan ser sustituidas por otras regiones cerebrales.

Si una burocracia representa menos de la suma de sus partes, una red equivale a muchas veces la suma de sus partes. Las redes constituyen una fuente de energía que la historia no había aprovechado hasta ahora:esos múltiples movimientos sociales autosuficientes, ligados entre si para conseguir una serie de objetivos, cuyo cumplimiento traería aparejada la transformación de todos los aspectos de la vida contemporánea.

Según Gerlach en un primer momento los antropólogos que se ocuparon de observar las redes, pensaban que estas carecían de líderes. En realidad dice "no hay escasez de líderes sino una gran profusión de éstos".

Para Hine los SPINS (Redes integradas politécnicas segmentadas) son hasta tal punto cualitativamente distintas de las burocracias en cuanto a organización e influencia, que la mayor parte de la gente no se percata de su existencia. A menudo las redes emprenden acciones similares sin ponerse de acuerdo previamente, simplemente a causa de las muchas convicciones que comparten. Podría también decirse que por el hecho de compartir esas convicciones están en connivencia.

Son infinitamente mas complejas que si fueran una red de pescar por su naturaleza tridimensional.

Otra comparación que puede o no satisfacernos según el conocimiento que tengamos del mismo es la del sistema nervioso, quizá es algo más que una metáfora pero conviene profundizar en ella por las analogías que pueda tener con la red. En cierto sentido el cerebro y el entramado de redes operan de forma muy similar. La estructura del cerebro es más afín a al idea de asociación que a la de jerarquía. "Lo que hace nacer los significa dos en el cerebro son patrones dinámicos, conexiones entre grupos de neuronas e interacciones entre estos grupos. La ener gía del cerebro está descentralizada. En los estados de con- 
ciencia caracterizados por una mayor expansión y coherencia es también donde la enrgía es mas amplia y ordenadamente dis ponible. El cerebro está entonces plenamente despierto. De igual forma, las redes son una forma alerta y reactiva de organi zación social. La información se mueve en ella de un forma no lineal, simultánea y significativa".

Probablemente influye en la concepción que se tiene de la red la representación gráfica que nos hacemos de ella, del mismo modo la analogía que hacemos con el sistema cerebral tal vez escape a nuestra comprensión o precise un acto de fe. En cualquier caso la representación no debe ser bidimensional pues da una idea muy limitada.

Digamos que la red se asemeja no tanto a una constelación de redes como a una galaxia de constelaciones en forma de red, lo cual añade complejidad al estudio de este fenómeno pero no evita tener una visión plana en la que es fácil caer de nuevo en la idea de jerarquía.

Estructuralmente lo más importante de una red es que cada individuo está en su centro. Esta es la diferencia básica que encontramos al estudiar este fenómeno comparando distintos autores. No existe un centro, como no existe un líder -como ya apuntábamos antes- hay multitud de centros y líderes, por ello no es el discurso de la importancia, a la secundiaredad, la accesoriedad... en función de las distancias, conexiones... etc.

Las redes no son igualitarias sólo porque cada miembro sea igual a otro. Al contrario, dado que las redes son diagonales y tridimensionales, intervienen en ellas personas de todos los niveles posibles. Lo que ocurre en una red es que los miembros se tratan como iguales porque lo que es importante es la información, el gran ecualizador.

Las redes son entes vivos no fosilizados que responden a las demandas y vacios de la sociedad civil. La redes siguen siendo fluidas $y$ abiertas

«Si observamos la rápida proliferación de las redes y percibimos su fuerza, podemos comprender el impulso que suponen para la transformación mundial. La red es una institución de nuestro tiempo: un sistema abierto, una estructura disipativa tan rica y coherente que se encuentra continuamente en estado de flujo, en un equilibrio de susceptible de reordenación continua, abierto indefiniblemente a la transformación. Esta forma orgánica de transfor- 
mación social es la mas adaptativa desde el punto de vista biológico, es más eficaz y más consciente que las estructuras jerárqui cas de la civilización moderna. La red es plástica y flexible».

Marilyn Ferguson

A su vez la red es paradógicamente a un tiempo íntima y expansiva. A diferencia de las organizaciones verticales, puede mantener su cualidad personal o local, aunque siga creciendo. No es preciso plantearse la elección entre un compromiso con la comunidad o un compromiso a escala global; ambos son posibles a un tiempo.

\section{La redistribución del poder}

Las redes suponen mas que una redistribución otra forma de ejercer el poder. Dentro de la jerarquía las decisiones se toman de arriba-abajo, lo cual no quiere decir que los grandes cambios sociales se produzcan al inversa desde abajo-arriba desde el ejercicio de poder de los miembros de una sociedad unidos.

El ejercicio del poder al que se nos tiene acostumbrados y que se nos intenta condicionar es el del voto, esto supone dejar en nuestras manos el elegir a quienes o con que ideología se va a "gobernar" nuestro país, -aún siendo gobernar una palabra que viene grande a nuestros gestores y contables-. Siendo que podemos modificar nuestra realidad sin esperar que sean otros quienes lo hagan por nosotros, eso precisa unas ideas claras y el concurso de otro sin dividuos que las compartan.

El cambio del poder vertical al horizontal que proporcionan las redes es liberador para los individuos. Las jerarquías favorecen el ascenso y el adelanto, produciendo stress, tensión y ansiedad.Las redes potencian al individuo y las personas que participan en ellas tienden a nutrirse unas a otras. En el entorno de la red la recompensa se produce al dar poder a los demás, no al pasar por encima de ellos.

Mientras muchas de nuestras instituciones se tambalean, una versión en nuestro siglo de la antigua tribu ha hecho ya su aparición: la red instrumento del paso siguiente de la evolución humana. La red redistribuye el poder. Amplificada por las comunicaciones electrónicas, y liberada de las antiguas restricciones 
familiares y culturales, la red es el antídoto de la alienación (para quienes quieren formar parte de ella). Genera la suficiente energía para remodelar la sociedad. Ofrece al individuo apoyo emocional, intelectual, epiritual y económico. Es un hogar invisible. Un medio poderoso de alterar el curso de las instituciones, especialmente del gobierno.

Como dice Theodor Roszak, los antiguos movimientos de masas revolucionarios no ofrecían a las personas mayor refugio que el que ofrecían las sociedades capitalistas. "Necesitamos una clase mas pequeña que el proletariado... La nueva política hablará a favor de millones... no uno a uno".

Una idea que se repite es el carácter liberador de las redes. José Argüelles las compara con la fuerza biológica de la sintro pía. "Esa tendencia de la energía vital hacia formas de asociación de comunicación y de conciencia siempre mayores. Las redes son tremendamente liberadoras el individuo es su centro..."

También se da en ellas un efecto de sinergia, ese plus de energía, resultado de una cooperación que tiene lugar en el seno de los sistemas naturales. Según vamos descubirendo ese efecto en las relaciones con los demás, en nuestro pequeño grupo, su efecto benéfico potencial para la sociedad resulta cada vez más evidente. Como dice el físico John Platt: "Siempre que las personas, aunque no sean mas que dos, comienzan a darse entre sí, o a trabajar los unos para los otros, inmediata mente aparecen esos resultados: ese mayor beneficio mutuo, ese mayor bienestar, y ese mayor desarrollo individual al mismo tiempo".

A la pregunta de qué pretenden las redes hay varias respuestas muy diferentes. No solo no hay redes que no sean iguales; cada una de por si cambia con el transcurso del tiempo, porque es un reflejo de las necesidades e intereses fluctuantes de sus miembros. Pero su objetivo esencial es redistribuir el poder.

¿Cual es el futuro de la red?: No estamos capacitados ara preverlo pero En una época en la que los valores descentralizadores y de establecimiento de redes está adquiriendo mayor aceptación y en que las organizaciones han de replantearse sus propósitos y sus valores mientras nos enfrentamos en el sector empresarial a un competencia sin precedentes, no es aconsejable ser un líder tradicional, sea social, sea político o empresarial. 
«Una forma no regresiva de recuperar cobijos, abrigos frente a la intemperie; de dar sentido a un mundo (social y natural) cuya creciente fragilidad y dislocamiento hoy hace despertar a tantos y tantas. Las gentes, hoy en día están aprendiendo a relacionarse (la sociedad está por reinventar) en una situación de aguda crisis. Este es el áspero terreno de juego donde se mueven los nuevos movimientos sociales, en las sociedades de la información, movimientos a veces descritos como redes de redes donde cooperan personas en una lucha desigual por la supervivencia y la emancipación. Redes, por tanto, que no sirven para apresar, sino que intentan -desde la mínima, desesperada, residual esperanza aún posible en el sombrío principio de siglo- abrir una perspectiva de justicia y libertad»

Francisco Fernandez Buey Jorge Riechmann

\section{Bibliografía}

van the BOGART, W. Information Network.

DABAS, E. y NAJMANOVICH, D. Redes. El lenguaje de los vín culos.

ERIKSON, E. Gandhi's Truth.

FERGUSON, M. The Acuarios Conspiracy.

FROMM, E. The sane society.

HINE, V. The basic paradigm of a Future Socio-cultural System.

LIPNACK y STAMPS. Networking.

McGREGOR, D. The human side of enterprise.

NAISBITT, J. Megatrends.

OUCHI, W. Theory $Z$.

REQUENA SANTOS, F. El concepto de red social, REIS.

RIECHMAN, J. y FERNÁNDEZ BUEY, F. Redes que dan libertad.

ROSZAK, T. El nacimiento de una contracultura.

TOFFLER, A. La Tercera Ola.

THOREAU, H.D. Civil Desobedience.

VVAA. Analisis de redes sociales, Política y Sociedad, ㄲo 33 . 